\title{
Three-dimensional frequency-domain full waveform inversion based on the nearly-analytic discrete method
}

\author{
DeYao Zhang ${ }^{1}$, WenYong Pan², DingHui Yang ${ }^{1 *}$, LingYun Qiu ${ }^{3}$, XingPeng Dong ${ }^{1}$, and WeiJuan Meng1 \\ 'Department of Mathematical Sciences, Tsinghua University, Beijing 100084, China; \\ 2Key Laboratory of Petroleum Resource Research, Institute of Geology and Geophysics, Chinese Academy of Sciences, Beijing 100029, China; \\ 3Yau Mathematical Sciences Center, Tsinghua University, Beijing 100084, China

\section{Key Points:} \\ - Nearly analytic discrete method is applied to solving 3D frequency-domain acoustic wave equation. \\ - Compared to traditional finite difference methods, the nearly analytic discrete method can solve the wave equation more accurately \\ and efficiently. \\ - Nearly analytic discrete method is more suitable than traditional methods for 3D frequency-domain full waveform inversion.
}

Citation: Zhang, D. Y., Pan, W. Y., Yang, D. H., Qiu, L. Y., Dong, X. P. and Meng, W. J. (2021). Three-dimensional frequency-domain full waveform inversion based on the nearly-analytic discrete method. Earth Planet. Phys., 5(2), 149-157. http://doi.org/10.26464/epp2021022

\begin{abstract}
The nearly analytic discrete (NAD) method is a kind of finite difference method with advantages of high accuracy and stability. Previous studies have investigated the NAD method for simulating wave propagation in the time-domain. This study applies the NAD method to solving three-dimensional (3D) acoustic wave equations in the frequency-domain. This forward modeling approach is then used as the "engine" for implementing 3D frequency-domain full waveform inversion (FWI). In the numerical modeling experiments, synthetic examples are first given to show the superiority of the NAD method in forward modeling compared with traditional finite difference methods. Synthetic 3D frequency-domain FWI experiments are then carried out to examine the effectiveness of the proposed methods. The inversion results show that the NAD method is more suitable than traditional methods, in terms of computational cost and stability, for 3D frequency-domain FWI, and represents an effective approach for inversion of subsurface model structures.
\end{abstract}

Keywords: three-dimension; frequency-domain; NAD method; forward modeling; full waveform inversion

\section{Introduction}

In recent decades, full waveform inversion (FWI) has become increasingly popular in global and exploration seismology due to its ability to provide high-resolution subsurface velocity structures (Lailly, 1983; Tarantola, 1986; Virieux and Operto, 2009). FWI can be performed both in the time domain (Mora, 1987; Tromp et al., 2005; Liu QY and Tromp, 2006; Tape et al., 2007; Bozdağ et al., 2016; Wang J et al., 2019; Dong XP et al., 2019) and the frequencydomain (Pratt and Worthington, 1990; Song and Williamson, 1995; Pratt, 1999; Operto et al., 2004; Sirgue and Pratt, 2004; Brossier et al., 2009). Compared with time-domain FWI, frequency-domain $\mathrm{FWl}$ is more flexible in selecting specific frequency components in a multi-scale inversion strategy. In addition, frequency-domain $\mathrm{FWI}$ is computationally more efficient, thanks to solving different sources simultaneously with one matrix factorization. However, in $3 \mathrm{D}$ frequency-domain $\mathrm{FWI}$ the matrix size grows exponentially (Operto et al., 2007; Plessix, 2009). The iterative solution of the seismic inverse problem requires a large number of forward simu-

Correspondence to: D. H. Yang, ydh@tsinghua.edu.cn

Received 30 SEP 2020; Accepted 27 JAN 2021.

Accepted article online 01 MAR 2021.

C 2021 by Earth and Planetary Physics. lations. Forward modeling of the seismic waves is considered as the "engine" of and foundation for the implementation of FWI. Therefore, determining an accurate and efficient forward modeling approach is necessary and essential if FWI experiments are to be practical.

In the past decades, researchers have studied a series of forward modeling methods, including finite difference methods (Kelly et al., 1976; Day, 1982; Igel et al., 1995; Yang DH et al., 2003, 2004, 2006; Liu SL et al., 2015, Ma X et al., 2018), finite element methods (Lysmer and Drake, 1972; Marfurt, 1984; Liu SL et al., 2014; He XJ et al., 2020), a pseudo-spectral method (Kosloff and Baysal, 1982), and a spectral element method (Kosloff and Baysal, 1982; Seriani and Priolo, 1994; Komatitsch et al., 2005). These methods are designed to solve different types of questions. Each may have superiorities in some aspects but limitations in others. Among these methods, finite difference methods are widely used because of the advantages of easy implementation, low computational cost, etc.

The nearly analytic discrete (NAD) method is a finite difference method that uses displacement and its gradient to approximate the high-order partial derivatives in the wave equation (Yang DH et al., 2003, 2004, 2006; Tong P et al., 2011, 2013). As this method 
captures more detailed wave equation information, dispersion analysis and numerical experiments have shown that it can solve the wave equation more accurately and efficiently than traditional finite difference methods (Yang DH et al., 2010, 2012). Previous studies have implemented the NAD method for forward modeling and reverse time migration in time-domain applications (Yang DH et al., 2003; Li JS et al., 2013). This study extends the NAD method to solve the three-dimension (3D) acoustic wave equation in the frequency-domain, which is then used as the "engine" for conducting 3D frequency-domain FWI. The paper is organized as follows. First, we introduce the 3D frequency-domain acoustic wave equation's discretization with a perfect matched layer (PML) absorbing boundary condition, which gives the linear system equations. According to the characteristics of the coefficient matrix (or "impedance matrix"), we choose the Krylov subspace iteration with incomplete LU decomposition to solve the linear system. We then introduce the theoretical basis of 3D FWI in the frequency-domain. The non-linear conjugate gradient method is adopted for model updating in the iterative inversion process. Finally, we give numerical examples of forward modeling and $3 \mathrm{D}$ frequency-domain FWI. The forward modeling experiments show that the NAD method for the 3D frequency-domain acoustic wave equation works well and can suppress the numerical dispersion effects more effectively than traditional finite difference methods. The FWI experiments show that the frequency-domain FWI with the NAD method can efficiently and stably reconstruct the subsurface velocity structures.

\section{Forward Modeling with the NAD Method in}

\section{Frequency-Domain}

In the frequency-domain, the 3D acoustic wave equation with constant density can be written as

$$
\Delta u(x, y, z)+\frac{\omega^{2}}{c^{2}} u(x, y, z)=-\frac{1}{c^{2}} s(x, y, z), \quad(x, y, z) \in D,
$$

where $u$ is pressure wavefield, $\Delta$ is Laplace operator, $\omega$ indicates angular frequency, $c=c(x, y, z)$ is the compressional velocity of the medium, $s$ is the seismic source term, and $D$ denotes the whole $3 \mathrm{D}$ computing area.

To discretize the acoustic wave equation with the NAD method, we need to derive the partial derivative form of the frequency-domain equation with respect to $x, y$ and $z$, respectively, as illustrated in the following equations:

$$
\left\{\begin{array}{l}
\frac{\partial^{2} u}{\partial x^{2}}+\frac{\partial^{2} u}{\partial y^{2}}+\frac{\partial^{2} u}{\partial z^{2}}+\frac{\omega^{2}}{c^{2}} u=-\frac{1}{c^{2}} s, \\
\frac{\partial^{2} u_{x}}{\partial x^{2}}+\frac{\partial^{2} u_{x}}{\partial y^{2}}+\frac{\partial^{2} u_{x}}{\partial z^{2}}+\frac{\omega^{2}}{c^{2}} u_{x}=-\frac{1}{c^{2}} \frac{\partial s}{\partial x^{\prime}} \\
\frac{\partial^{2} u_{y}}{\partial x^{2}}+\frac{\partial^{2} u_{y}}{\partial y^{2}}+\frac{\partial^{2} u_{y}}{\partial z^{2}}+\frac{\omega^{2}}{c^{2}} u_{y}=-\frac{1}{c^{2}} \frac{\partial s}{\partial y^{\prime}} \\
\frac{\partial^{2} u_{z}}{\partial x^{2}}+\frac{\partial^{2} u_{z}}{\partial y^{2}}+\frac{\partial^{2} u_{z}}{\partial z^{2}}+\frac{\omega^{2}}{c^{2}} u_{z}=-\frac{1}{c^{2}} \frac{\partial s}{\partial z} .
\end{array}\right.
$$

Second, we apply the PML condition (Komatitsch and Tromp, 2003), which is widely used in the area of seismic wave propagation, in Equation (2) for eliminating the reflections from artificial boundaries. The real coordinate $x$ in the original differential equa- tions is replaced with the complex coordinate $\tilde{x}$ in the PML region. The relation between $x$ and $\tilde{x}$ is:

$$
\tilde{x}=x-\frac{\mathrm{i}}{w} \int_{0}^{x} d_{x}(s) \mathrm{d} s,
$$

where $d_{x}(s)>0$ is an attenuation function, and $i$ denotes the imaginary unit. We choose $d s:=d_{x}(s)$ as (Komatitsch and Tromp, 2003):

$$
d_{x}(s)=-\frac{3 c}{2 l} \log (R)\left(\frac{s}{l}\right)^{2} .
$$

In Equation (4), c is the wave propagation velocity between the PML region and real physical region, I indicates the depth of the PML region, $s$ is the position in the PML layer, and $R$ is theoretical reflection parameter which can be very small (in our models, it is set as 0.001).

In the PML region, after replacing $x, y$ and $z$ with $\tilde{x}, \tilde{y}$ and $z$, Equation (2) can be rewritten as:

$$
\left\{\begin{array}{l}
\frac{\partial^{2} u}{\partial \tilde{x}^{2}}+\frac{\partial^{2} u}{\partial \tilde{y}^{2}}+\frac{\partial^{2} u}{\partial \tilde{z}^{2}}+\frac{\omega^{2}}{c^{2}} u=-\frac{1}{c^{2}} s, \\
\frac{\partial^{2} u_{\tilde{x}}}{\partial \tilde{x}^{2}}+\frac{\partial^{2} u_{\tilde{x}}}{\partial \tilde{y}^{2}}+\frac{\partial^{2} u_{\tilde{x}}}{\partial \tilde{z}^{2}}+\frac{\omega^{2}}{c^{2}} u_{\tilde{x}}=-\frac{1}{c^{2}} \frac{\partial s}{\partial \tilde{x}^{\prime}} \\
\frac{\partial^{2} u_{\tilde{y}}}{\partial \tilde{x}^{2}}+\frac{\partial^{2} u_{\tilde{y}}}{\partial \tilde{y}^{2}}+\frac{\partial^{2} u_{\tilde{y}}}{\partial \tilde{z}^{2}}+\frac{\omega^{2}}{c^{2}} u_{\tilde{y}}=-\frac{1}{c^{2}} \frac{\partial s}{\partial \tilde{y}^{\prime}} \\
\frac{\partial^{2} u_{\tilde{z}}}{\partial \tilde{x}^{2}}+\frac{\partial^{2} u_{\tilde{z}}}{\partial \tilde{y}^{2}}+\frac{\partial^{2} u_{\tilde{z}}}{\partial \tilde{z}^{2}}+\frac{\omega^{2}}{c^{2}} u_{\tilde{z}}=-\frac{1}{c^{2}} \frac{\partial s}{\partial \tilde{z}} .
\end{array}\right.
$$

Then we turn the variables $\tilde{x}, \tilde{y}$ and $\tilde{z}$ in Equation (5) to $x, y$ and $z$. By referring to Equation (3), we see that the derivative can be computed as

$$
\frac{\partial x}{\partial \tilde{x}}=\frac{\mathrm{i} \omega}{\mathrm{i} \omega+d_{x}} .
$$

Applying chain rule to Equation (5), taking $\tilde{x}$ as an example, the result is given in the following equation:

$$
\left\{\begin{array}{l}
\frac{\partial u}{\partial \tilde{x}}=\frac{\mathrm{i} \omega}{\mathrm{i} \omega+d_{x}} \frac{\partial u}{\partial x^{\prime}} \\
\frac{\partial^{2} u}{\partial \tilde{x}^{2}}=\left(\frac{\mathrm{i} \omega}{\mathrm{i} \omega+d_{x}}\right)^{2} \frac{\partial^{2} u}{\partial x^{2}}-\frac{(\mathrm{i} \omega)^{2} d_{x p}^{\prime}}{\left(\mathrm{i} \omega+d_{x}\right)^{3}} \frac{\partial u}{\partial x^{\prime}} \\
\frac{\partial^{3} u}{\partial \tilde{x}^{3}}=\left(\frac{\mathrm{i} \omega}{\mathrm{i} \omega+d_{x}}\right)^{3} \frac{\partial^{3} u}{\partial x^{3}}-\frac{3(\mathrm{i} \omega)^{3} d_{x p}^{\prime}}{\left(\mathrm{i} \omega+d_{x}\right)^{4}} \frac{\partial^{2} u}{\partial x^{2}}+\left(\frac{3(\mathrm{i} \omega)^{3} d_{x p}^{\prime 2}}{\left(\mathrm{i} \omega+d_{x}\right)^{5}}-\frac{(\mathrm{i} \omega)^{3} d_{x p p}^{\prime \prime}}{\left(\mathrm{i} \omega+d_{x}\right)^{4}}\right) \frac{\partial u}{\partial x^{\prime}} \\
\frac{\partial^{3} u}{\partial \tilde{y}^{2} \partial \tilde{x}}=\frac{(\mathrm{i} \omega)^{3}}{\left(\mathrm{i} \omega+d_{x}\right)\left(\mathrm{i} \omega+d_{y}\right)^{2}} \frac{\partial^{3} u}{\partial y^{2} \partial x}-\frac{(\mathrm{i} \omega)^{3} d_{y p}^{\prime}}{\left(\mathrm{i} \omega+d_{x}\right)\left(\mathrm{i} \omega+d_{y}\right)^{3}} \frac{\partial^{2} u}{\partial y \partial x^{\prime}}
\end{array}\right.
$$

where $d_{x p}^{\prime}$ and $d_{x p p}^{\prime \prime}$ are the first-order and second-order derivatives of $d_{x}$. We can replace $\tilde{x}, \tilde{y}$ and $\tilde{z}$ to obtain the corresponding partial derivatives, then apply all of the partial derivatives to Equation (5). The final 3D acoustic wave equation of the NAD method with PML boundary condition is obtained. The whole discretized wave equation is illustrated in the Appendix. In the end, we apply the fourth-order NAD method to discretize the 3D wave equation following Lang $C$ and Yang DH (2017). After discretization with the fourth-order NAD method, the 3D frequency-domain acoustic wave equation can be written in compact matrix form, known as a linear system equation:

$$
A U=s,
$$


where $\boldsymbol{A}$ is the impedance matrix, $\boldsymbol{U}$ is the pressure wavefield vector, and $s$ is the source term vector. In the NAD method, the wavefield includes its spatial derivatives $\boldsymbol{U}=\left(u, u_{x}, u_{y}, u_{z}\right)$. Lang $C$ and Yang DH (2017) have carried out many studies concerning solutions of Equation (8). In this study, to solve the linear system we use the Krylov subspace iteration (GMRES and BiCGSTAB iteration) with incomplete LU (ILU) decomposition precondition. A Ricker wavelet is used as the source time function:

$$
w(t)=A\left[1-2\left(\pi f_{0} t\right)^{2}\right] e^{-\left(\pi f_{0} t\right)^{2}}
$$

where $A$ is the amplitude of seismic source, $f_{0}$ denotes the central frequency, and $e$ is the Euler number. In the frequency-domain, the source wavelet is obtained as:

$$
F[w(t)]=\frac{\sqrt{2} A}{\pi f_{0}}\left(\frac{f}{f_{0}}\right)^{2} e^{-\left(\frac{f}{f_{0}}\right)^{2}},
$$

where $f$ denotes frequency.

\section{Full Waveform Inversion}

In this section, the theory of 3D frequency-domain FWI based on the NAD method is introduced. In FWI, the model properties are updated iteratively by minimizing an objective function that measures the observed and synthetic seismic data differences. The L-2 norm objective function is formulated as:

$$
E(c)=\frac{1}{2} \sum_{\omega, i}\left[\delta d^{i}(c, \omega)\right]^{\mathrm{H}} \delta d^{i}(c, \omega),
$$

where $(\cdot)^{\mathrm{H}}$ denotes the conjugate transpose of the matrix, and $\delta d^{i}$ is the data residual at the $i$-th source, which is obtained by

$$
\delta d_{j}^{i}(c, \omega)=u_{j}^{i}(c, \omega)-d_{j}^{i}\left(c_{\text {true }}, \omega\right), \quad j=1,2, \cdots, n,
$$

where $c_{\text {true }}$ means the real model (or target model), $n$ denotes the total number of receivers, $d_{j}^{i}\left(c_{\text {true }}, \omega\right)$ is the observed data recorded on the $j$-th receiver of the $i$-th source, and $u_{j}^{i}(c, \omega)$ is the corresponding synthetic data.

The full waveform inverse problem is commonly solved within the gradient-based optimization framework. Different local optimization methods, including steepest descent, non-linear conjugate gradient (NCG), quasi-Newton methods, etc. (Nocedal and Wright, 2006; Pan WY et al., 2017), can be used for the model updating. Within these optimization methods, the first-order derivative of the misfit function, namely the gradient, needs to be calculated first. Following Pratt (1999), the gradient of the misfit function $E(c)$ can be written as:

$$
\nabla E(c)=\frac{\partial E}{\partial c}=\operatorname{Re}\left(J^{\top} \delta \boldsymbol{d}^{*}\right)
$$

where $\boldsymbol{J}$ is the partial derivative of the synthetic wavefield data $\boldsymbol{U}=\left(u_{1}, u_{2}, \cdots, u_{n}\right)^{\top}\left((\cdot)^{\top}\right.$ and $(\cdot)^{*}$ mean the transpose and conjugate of vectors):

$$
\boldsymbol{J}=\frac{\partial \boldsymbol{U}}{\partial c}=\left(\begin{array}{cccc}
\frac{\partial u_{1}}{\partial c_{1}} & \frac{\partial u_{1}}{\partial c_{2}} & \cdots & \frac{\partial u_{1}}{\partial c_{m}} \\
\vdots & \vdots & \vdots & \vdots \\
\frac{\partial u_{n}}{\partial c_{1}} & \frac{\partial u_{n}}{\partial c_{2}} & \cdots & \frac{\partial u_{n}}{\partial c_{m}}
\end{array}\right)=\left[\begin{array}{llll}
\frac{\partial u}{\partial c_{1}} & \frac{\partial u}{\partial c_{2}} & \cdots & \frac{\partial u}{\partial c_{m}}
\end{array}\right]
$$

However, it is computationally unaffordable to calculate the partial derivative wavefield explicitly for large-scale inverse problems. With the help of the linear system in Equation (8), the partial derivative wavefield can be derived as (Pratt et al., 1998; Pan WY et al., 2016):

$$
\frac{\partial U}{\partial c}=-A^{-1} \frac{\partial A}{\partial c} U
$$

Substituting Equation (15) into Equation (14) then into Equation (13), we can obtain the gradient as:

$$
\nabla E(c)=-\operatorname{Re}\left[\boldsymbol{U}^{\top}\left(\frac{\partial \boldsymbol{A}}{\partial c}\right)^{\top} \boldsymbol{A}^{-\top} \delta \boldsymbol{d}^{*}\right]
$$

where $\frac{\partial \boldsymbol{A}}{\partial \boldsymbol{c}}, \boldsymbol{U}$ and $\delta \boldsymbol{d}^{*}$ can be obtained easily, but for large models the cost of calculating $A^{-\top}$ directly is impracticable. Instead, the gradient can be computed efficiently by cross-correlating the forward and adjoint wavefields:

$$
\nabla E(c)=-\operatorname{Re}\left[\boldsymbol{U}^{\top}\left(\frac{\partial \boldsymbol{A}}{\partial c}\right)^{\top} \boldsymbol{v}\right]
$$

where $\boldsymbol{v}$ is known as the adjoint wavefield obtained by solving the following adjoint wave equation:

$$
\boldsymbol{A}^{\top} \boldsymbol{v}=\delta \boldsymbol{d}^{*}
$$

The LU decompositions of $\boldsymbol{A}$ and $\boldsymbol{A}^{\top}$ are similar. In the previous section, we have obtained the ILU decomposition of $\boldsymbol{A}$. Thus, Equation (19) can be solved using the same approach with the fourth-order NAD method. At each non-linear iteration, the model parameter $m$ can be updated by

$$
m_{k+1}=m_{k}+a_{k} \delta m_{k}
$$

where $a_{k}$ and $\delta m_{k}$ are the step length and search direction at the $k$-th iteration. The step length is calculated using the line search method. In this study, we adopt the NCG method for solving the inverse problem. The search direction is calculated by

$$
\delta m_{k}=-\nabla E_{k}+\beta_{k} \delta m_{k-1}
$$

where $\beta_{k}$ is a scalar such that $\delta m_{k}$ and $\delta m_{k-1}$ are conjugate. It can be obtained with the "Fletcher-Reeves", "Polak-Ribiere", "Hestenes-Stiefel" or "Dai-Yuan" methods.

\section{Numerical Experiments}

This section first gives two forward modeling examples to illustrate the NAD method's advantages compared to traditional finite difference methods. We then present numerical examples of $3 \mathrm{D}$ frequency-domain FWI based on the NAD method.

\subsection{Homogeneous Model Example}

The 3D homogeneous model has a size of $4 \mathrm{~km}, 0.5 \mathrm{~km}$, and $4 \mathrm{~km}$ in $x, y$ and $z$ directions. The grid spacing is $25 \mathrm{~m}$. PML layers with a thickness of 6 are applied on all boundaries of the model for absorbing the reflections of artificial boundaries. The compressional velocity of the medium is $2 \mathrm{~km} / \mathrm{s}$. A Ricker wavelet with a dominant frequency of $30 \mathrm{~Hz}$, located at the center of the model, is used as the source for forward modeling.

Figure 1 shows the frequency-domain wavefield snapshot at the 
slice of $y=0.25 \mathrm{~km}$ with a frequency of $30 \mathrm{~Hz}$. Figure 2 shows the frequency-domain wavefield snapshots in 3D with a frequency of $11 \mathrm{~Hz}$. Figures 3, 4 and 5 are the wavefield snapshots in the time domain. In Figure 3, at the time of $t_{2}=1.233 \mathrm{~s}$, the wavefronts have spread out of the computation region without evident reflections, indicating that the PML boundary condition works effectively. However, it can be seen in Figure 5 that the fourth-order NAD method works much better in suppressing the numerical dis-

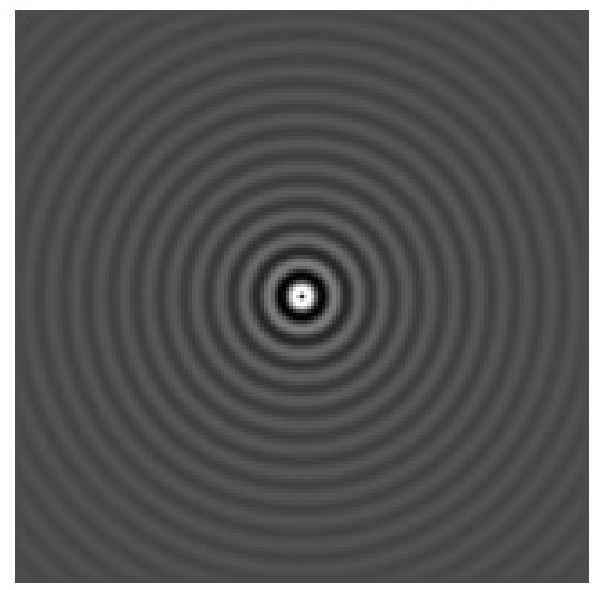

Figure 1. Wavefield snapshot with the frequency $f=30 \mathrm{~Hz}$.

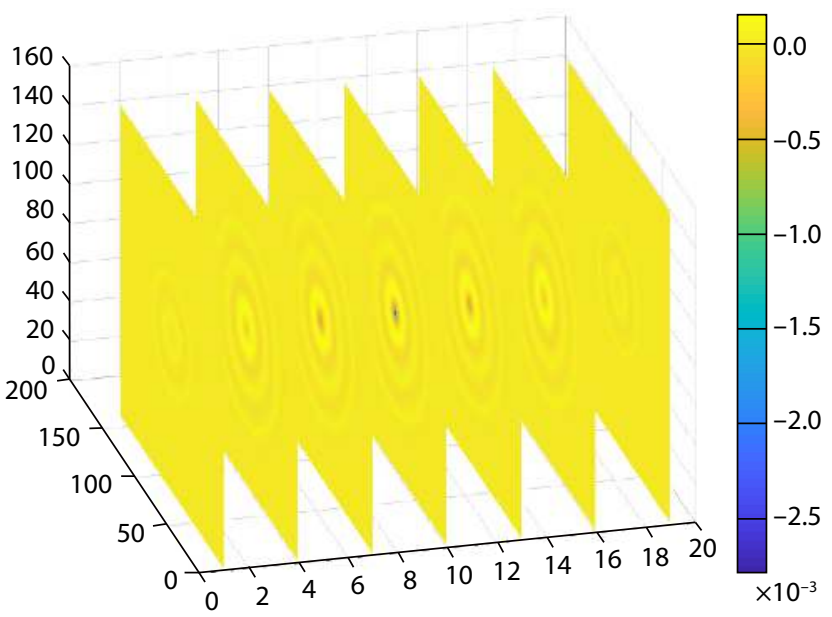

Figure 2. Wavefield snapshots with the frequency $f=11 \mathrm{~Hz}$.

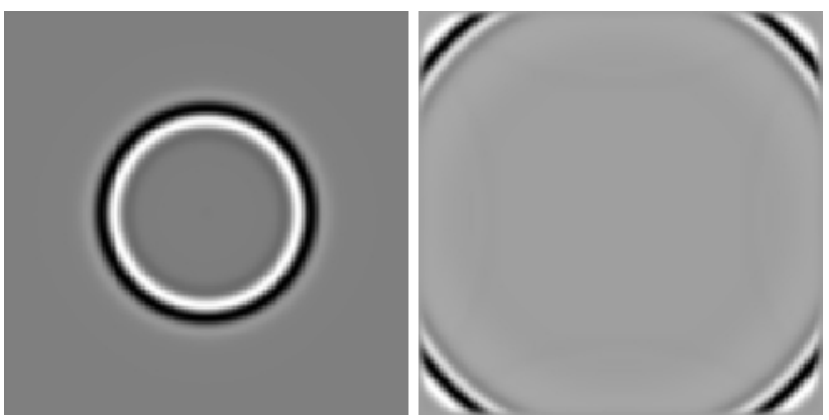

Figure 3. Time-domain wavefield snapshots at the times of $t_{1}=0.667$ $s$ (left), $t_{2}=1.233 \mathrm{~s}$ (right). persion effects, as indicated by the red boxes, than the traditional fourth-order finite difference method. Theory regarding suppression of dispersion effects is discussed in Lang $\mathrm{C}$ and Yang $\mathrm{DH}$ (2017).

\subsection{Two-layer Model Example}

Next, we test the NAD method with a two-layer model example to examine its effectiveness in handling internal discontinuity within the velocity structure. The model size, grid spacing, boundary conditions, and source configurations are all the same as those of the previous homogeneous model example. The interface is located at $2.7 \mathrm{~km}$ in depth. The upper and lower layers have velocities of $2 \mathrm{~km} / \mathrm{s}$ and $2.5 \mathrm{~km} / \mathrm{s}$, respectively. The internal discontinuity is described by velocity variation in the impedance matrix of Equation (8). Figures 6 and 7 show the wavefield snapshots calculated using the fourth-order NAD method in the frequency and time domains, respectively. As can be seen, the wavefields can be modeled accurately with few dispersion artifacts and boundary reflections. These observations in the numerical experiments verify the effectiveness and accuracy of the NAD method in forward modeling with internal discontinuity.

\subsection{Two-layer Model FWI Example}

To illustrate the effectiveness of our methods for frequency-domain FWI, we first carry out inversion experiments with a simple

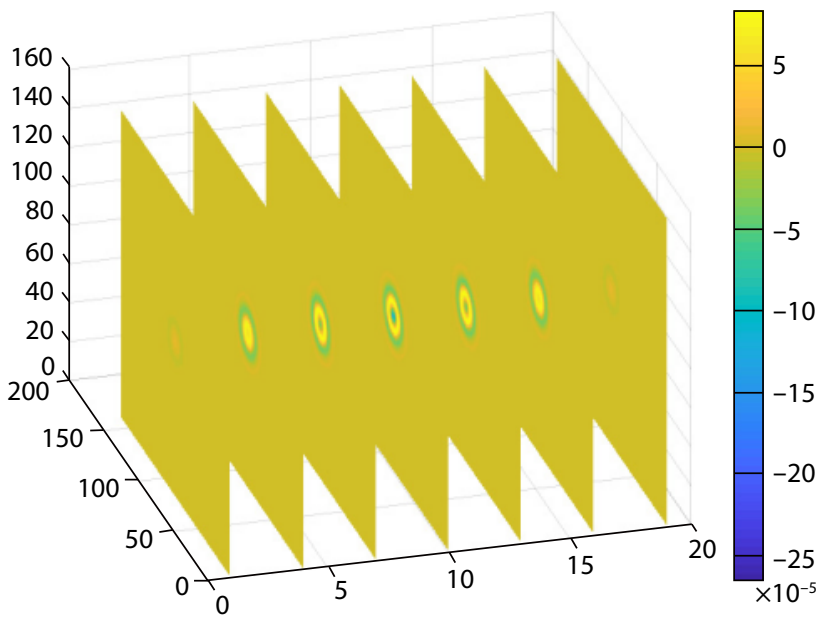

Figure 4. Time-domain wavefield snapshot at the time $t=0.267 \mathrm{~s}$.

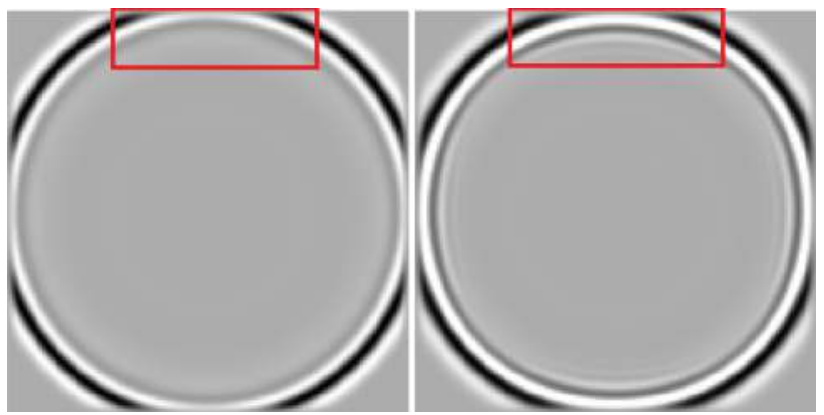

Figure 5. Time-domain wavefield snapshots obtained by the NAD method (left) and traditional finite difference method (right). 


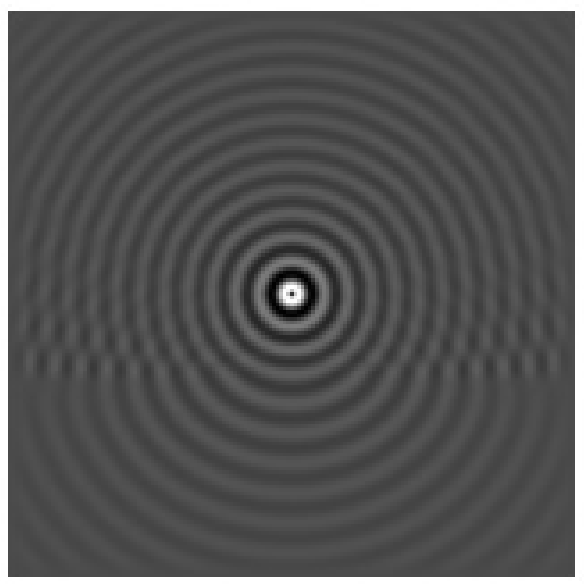

Figure 6. Frequency-domain snapshot with the frequency $f=30 \mathrm{~Hz}$.

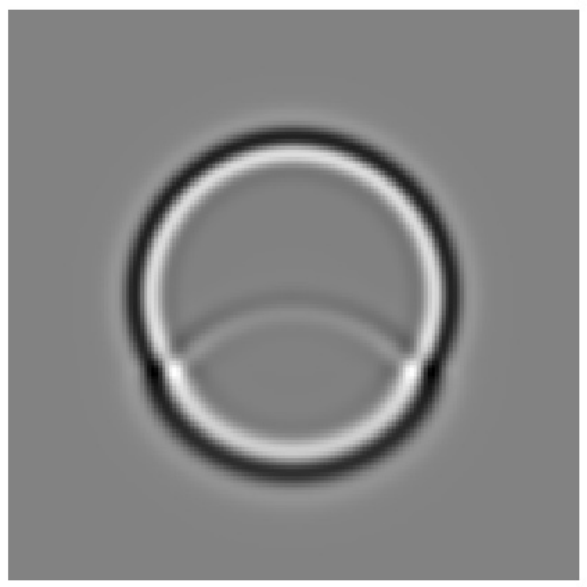

Figure 7. Time-domain snapshot at $t=0.667 \mathrm{~s}$.

two-layer model. The model sizes in $x, y$ and $z$ directions are $2.5 \mathrm{~km}, 0.5 \mathrm{~km}$, and $2.5 \mathrm{~km}$, respectively. The grid spacing is $25 \mathrm{~m}$. The number of grids in $x, y$ and $z$ directions are 101, 21, and 101. Figure 8 presents the target velocity model consisting of two layers. The upper layer has a velocity of $2.0 \mathrm{~km} / \mathrm{s}$ and the lower layer has a higher velocity of $2.5 \mathrm{~km} / \mathrm{s}$. The initial model is homogeneous with a constant velocity of $2.0 \mathrm{~km} / \mathrm{s}$. Three wells are placed in the middle of three sides of the model. Eleven sources and 89 receivers are deployed regularly in each well. A Ricker wavelet with dominant frequency of $15 \mathrm{~Hz}$ is used as the source function. We carry out the inversion experiments with a multi-scale strategy by ranging the frequency from $1 \mathrm{~Hz}$ to $21 \mathrm{~Hz}$.

Figure 9 shows the inversion result after 60 NCG iterations. The boundary between higher and lower velocity layers can be observed clearly. But the velocities in deeper parts are not well recovered. Figure 10 shows the final inversion result after 100 NCG iterations. As can be seen, the boundary of the velocity structure is obtained more accurately. But the velocities around the bottom edge are still not recovered very well. According to our experience, this may be caused by the settings of sources and receivers. The wave in the route from source to receivers cannot reach the bottom in the three wells.

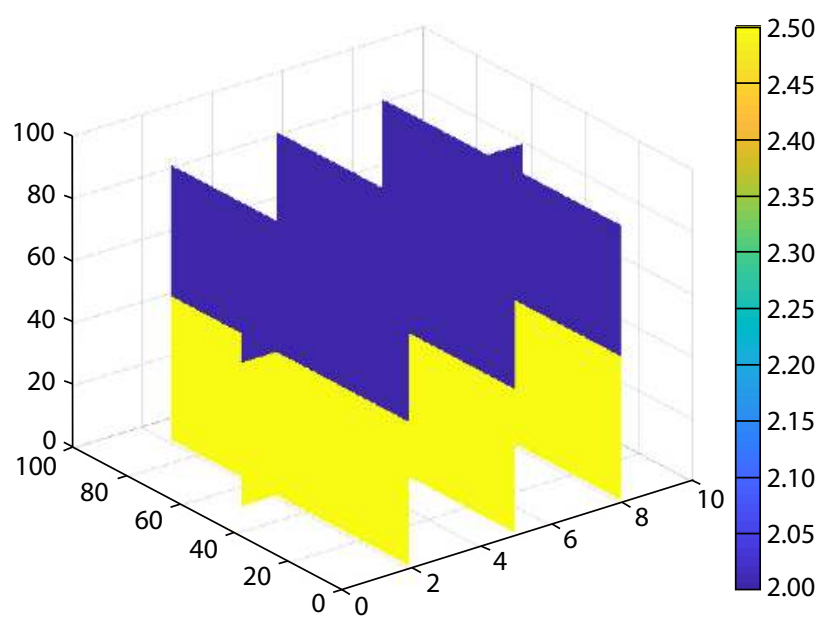

Figure 8. Two-layer true model.

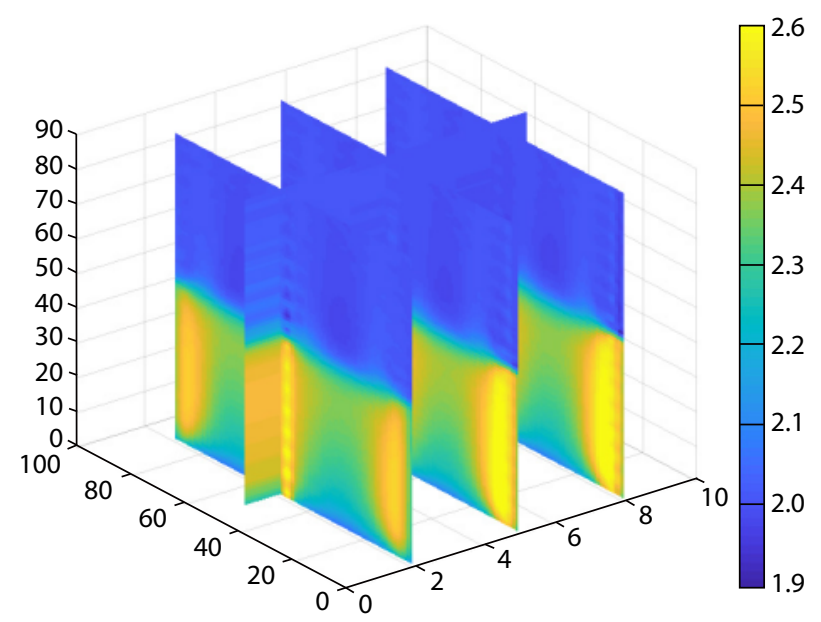

Figure 9. The inverted velocity model after 60 NCG iterations.

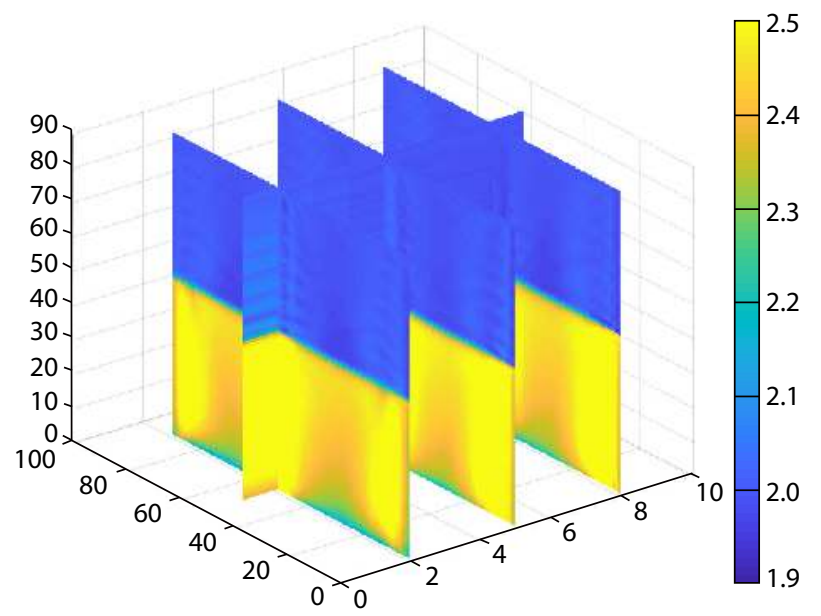

Figure 10. The inverted velocity model after 100 NCG iterations.

\subsection{SEG/EAGE 3D Overthrust Model Inversion Example}

Finally, we apply the frequency-domain FWI with the NAD method to a subsection of the SEG/EAGE 3D Overthrust model, which 
contains more complex velocity variations. The model has a size of $1.65 \mathrm{~km}, 2.55 \mathrm{~km}$, and $1.65 \mathrm{~km}$ in the $x, y$ and $z$ directions, respectively. The grid spacing is $50 \mathrm{~m}$. PML layers are applied to all boundaries of the model. Figures 11 and 12 present the 3D true and initial velocity models, respectively. In the $x$ direction, we arrange five wells on both sides regularly. Twenty-one sources and 43 receivers are deployed regularly in each well. The source function is a Ricker wavelet with dominant frequency of $15 \mathrm{~Hz}$. We choose 20 frequencies from $1 \mathrm{~Hz}$ to $31 \mathrm{~Hz}$ for inversion.

Figure 13 shows the inversion result after 100 NCG iterations. As can be seen, the velocity structures are accurately reconstructed. The structures in the bottom are also well inverted. These observations suggest that our NAD method works well for 3D frequency-domain FWI and can invert subsurface model structures accurately and stably.

\section{Conclusions}

This paper develops a nearly-analytic discrete method (NAD) method for $3 D$ acoustic wave equation forward modeling in the frequency-domain. We derive partial derivatives of the frequency- domain acoustic wave equation to obtain discretized NAD equations. The PML absorbing boundary condition is introduced into the system to absorb reflected waves from artificial boundaries. We then present the basic principle of FWI in the frequency-domain. The NAD method is applied in 3D frequency-domain FWI as a forward modeling "engine". Numerical examples are given to show that the NAD method works better than traditional finite difference methods. The proposed methods also work well for 3D frequency-domain FWI and can reconstruct model properties effectively.

\section{Acknowledgments}

This work was supported by the Joint Fund of Seismological Science (Grant No. U1839206) and the National R\&D Program on Monitoring, Early Warning and Prevention of Major Natural Disaster (Grant No. 2017YFC1500301). W. Pan is supported by IGGCAS Research Start-up Funds (Grant No. E0515402) and National Natural Science Foundation of China (Grant No. E1115401). L. Qiu's research is supported by National Natural Science Foundation of China (Grant No. 11971258).
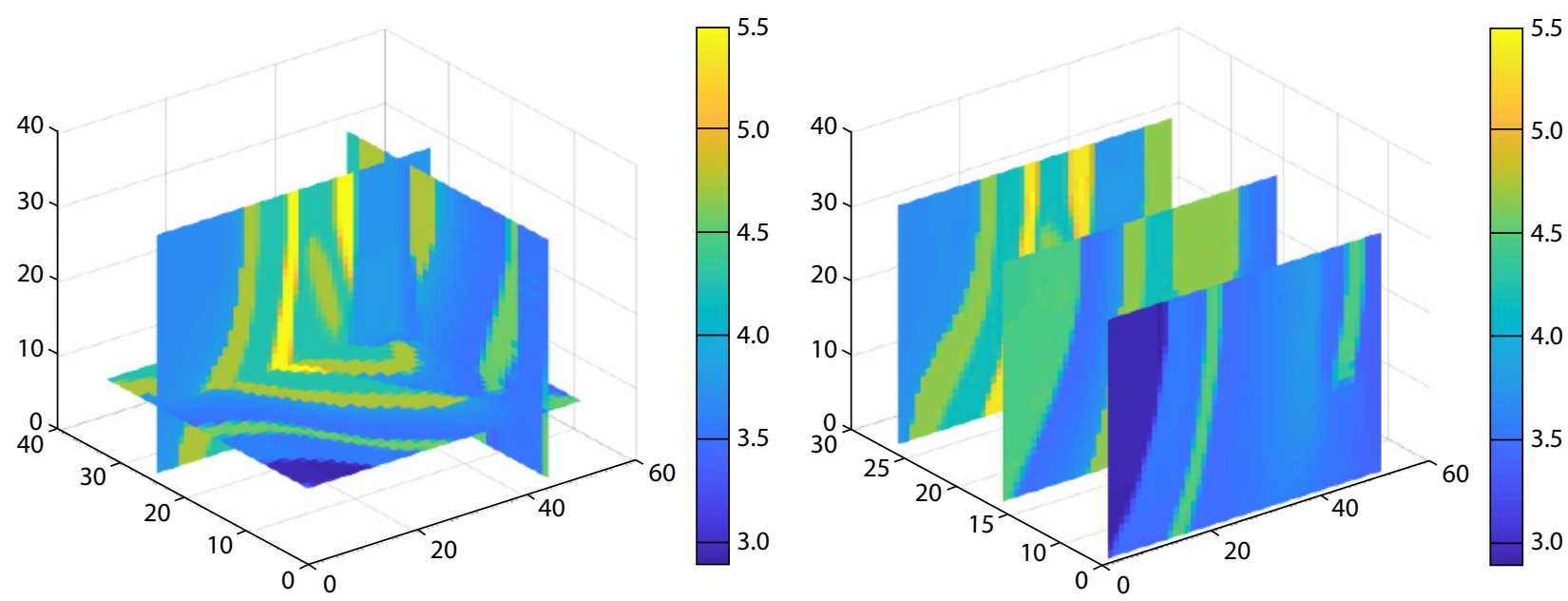

Figure 11. The true velocity model.
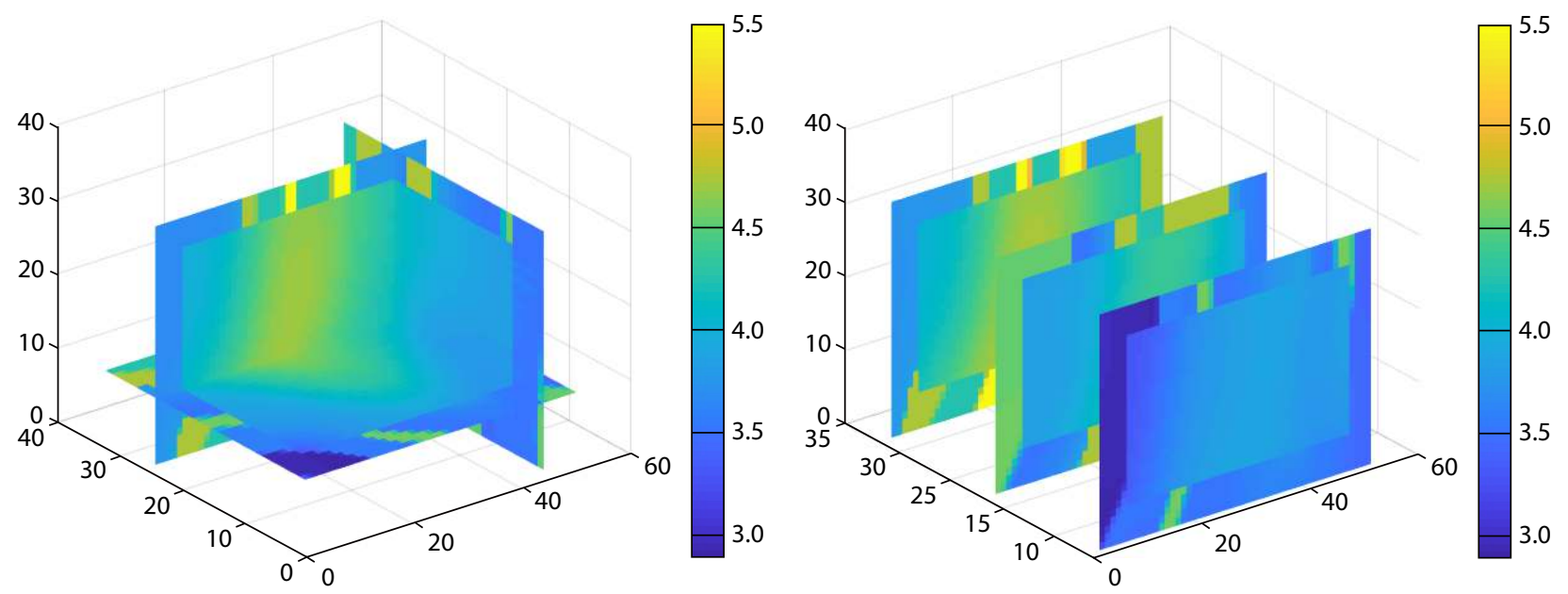

Figure 12. The initial velocity model. 

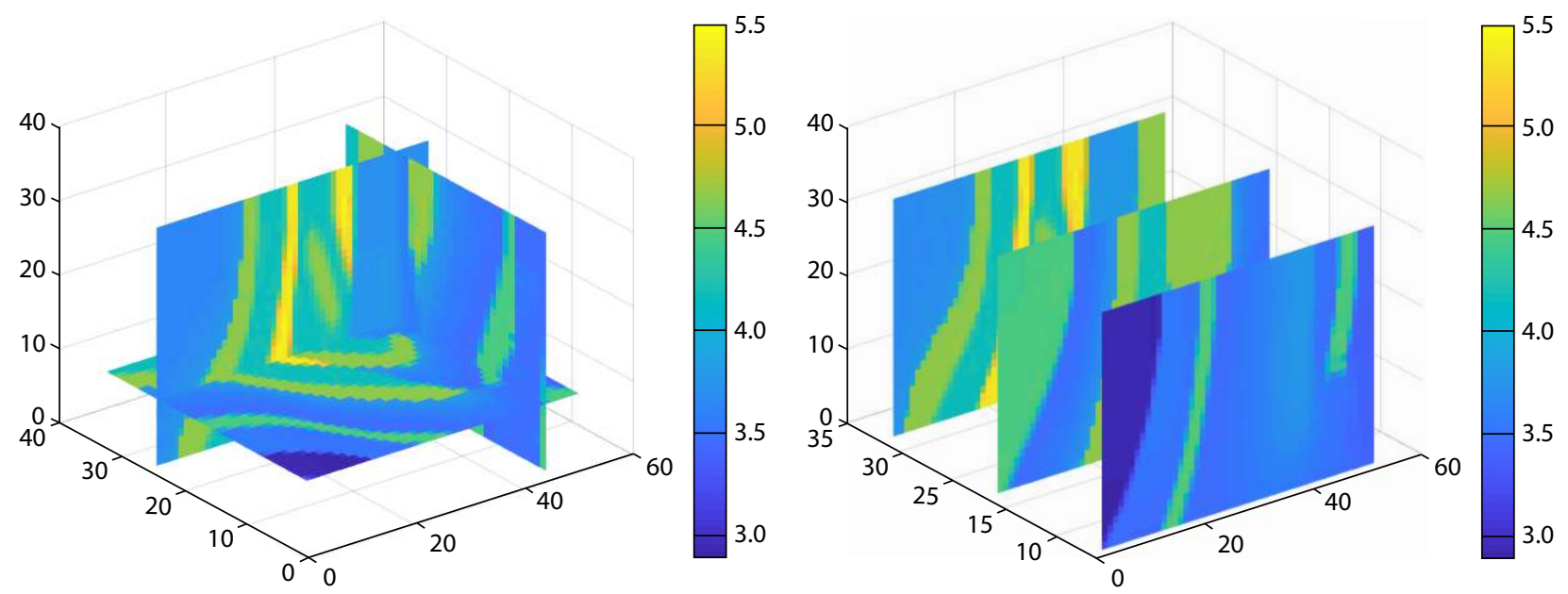

Figure 13. The inverted velocity model by frequency-domain FWI.

\section{Appendix: 3D Fourth-order NAD method}

Replacing the parameters in Equation (5) with Expressions (7) in $x, y$ and $z$ directions gives:

$$
\begin{aligned}
& \int\left(\frac{\mathrm{i} \omega}{\mathrm{i} \omega+d_{x}}\right)^{2} \frac{\partial^{2} u}{\partial x^{2}}+\left(\frac{\mathrm{i} \omega}{\mathrm{i} \omega+d_{y}}\right)^{2} \frac{\partial^{2} u}{\partial y^{2}}+\left(\frac{\mathrm{i} \omega}{\mathrm{i} \omega+d_{z}}\right)^{2} \frac{\partial^{2} u}{\partial z^{2}}- \\
& \frac{(\mathrm{i} \omega)^{2} d_{x p}^{\prime}}{\left(\mathrm{i} \omega+d_{x}\right)^{3}} \frac{\partial u}{\partial x}-\frac{(\mathrm{i} \omega)^{2} d_{y p}^{\prime}}{\left(\mathrm{i} \omega+d_{y}\right)^{3}} \frac{\partial u}{\partial y}-\frac{(\mathrm{i} \omega)^{2} d_{z p}^{\prime}}{\left(\mathrm{i} \omega+d_{z}\right)^{3}} \frac{\partial u}{\partial z}+\frac{\omega^{2}}{c^{2}} u=-\frac{1}{c^{2}} s, \\
& \left(\frac{\mathrm{i} \omega}{\mathrm{i} \omega+d_{x}}\right)^{2} \frac{\partial^{3} u}{\partial x^{3}}+\left(\frac{\mathrm{i} \omega}{\mathrm{i} \omega+d_{y}}\right)^{2} \frac{\partial^{3} u}{\partial y^{2} \partial x}+\left(\frac{\mathrm{i} \omega}{\mathrm{i} \omega+d_{z}}\right)^{2} \frac{\partial^{3} u}{\partial z^{2} \partial x}-\frac{3(\mathrm{i} \omega)^{2} d_{x p}^{\prime}}{\left(\mathrm{i} \omega+d_{x}\right)^{3}} \frac{\partial^{2} u}{\partial x^{2}}- \\
& \frac{(\mathrm{i} \omega)^{2} d_{y p}^{\prime}}{\left(\mathrm{i} \omega+d_{y}\right)^{3}} \frac{\partial^{2} u}{\partial y \partial x}-\frac{(\mathrm{i} \omega)^{2} d_{z p}^{\prime}}{\left(\mathrm{i} \omega+d_{z}\right)^{3}} \frac{\partial^{2} u}{\partial z \partial x}+\left(\frac{3(\mathrm{i} \omega)^{2} d_{x p}^{\prime 2}}{\left(\mathrm{i} \omega+d_{x}\right)^{4}}-\frac{(\mathrm{i} \omega)^{2} d_{x p p}^{\prime \prime}}{\left(\mathrm{i} \omega+d_{x}\right)^{3}}+\frac{\omega^{2}}{c^{2}}\right) \frac{\partial u}{\partial x}=-\frac{1}{c^{2}} \frac{\partial s}{\partial x^{\prime}}, \\
& \left(\frac{\mathrm{i} \omega}{\mathrm{i} \omega+d_{x}}\right)^{2} \frac{\partial^{3} u}{\partial x^{3}}+\left(\frac{\mathrm{i} \omega}{\mathrm{i} \omega+d_{y}}\right)^{2} \frac{\partial^{3} u}{\partial y^{2} \partial x}+\left(\frac{\mathrm{i} \omega}{\mathrm{i} \omega+d_{z}}\right)^{2} \frac{\partial^{3} u}{\partial z^{2} \partial x}-\frac{3(\mathrm{i} \omega)^{2} d_{y p}^{\prime}}{\left(\mathrm{i} \omega+d_{x}\right)^{3}} \frac{\partial^{2} u}{\partial x^{2}}- \\
& \frac{(\mathrm{i} \omega)^{2} d_{x p}^{\prime}}{\left(\mathrm{i} \omega+d_{y}\right)^{3}} \frac{\partial^{2} u}{\partial y \partial x}-\frac{(\mathrm{i} \omega)^{2} d_{z p}^{\prime}}{\left(\mathrm{i} \omega+d_{z}\right)^{3}} \frac{\partial^{2} u}{\partial z \partial x}+\left(\frac{3(\mathrm{i} \omega)^{2} d_{y p}^{\prime 2}}{\left(\mathrm{i} \omega+d_{x}\right)^{4}}-\frac{(\mathrm{i} \omega)^{2} d_{y p p}^{\prime \prime}}{\left(\mathrm{i} \omega+d_{x}\right)^{3}}+\frac{\omega^{2}}{c^{2}}\right) \frac{\partial u}{\partial x}=-\frac{1}{c^{2}} \frac{\partial s}{\partial x}, \\
& \left(\frac{\mathrm{i} \omega}{\mathrm{i} \omega+d_{x}}\right)^{2} \frac{\partial^{3} u}{\partial x^{3}}+\left(\frac{\mathrm{i} \omega}{\mathrm{i} \omega+d_{y}}\right)^{2} \frac{\partial^{3} u}{\partial y^{2} \partial x}+\left(\frac{\mathrm{i} \omega}{\mathrm{i} \omega+d_{z}}\right)^{2} \frac{\partial^{3} u}{\partial z^{2} \partial x}-\frac{3(\mathrm{i} \omega)^{2} d_{z p}^{\prime}}{\left(\mathrm{i} \omega+d_{x}\right)^{3}} \frac{\partial^{2} u}{\partial x^{2}}- \\
& \frac{(\mathrm{i} \omega)^{2} d_{y p}^{\prime}}{\left(\mathrm{i} \omega+d_{y}\right)^{3}} \frac{\partial^{2} u}{\partial y \partial x}-\frac{(\mathrm{i} \omega)^{2} d_{x p}^{\prime}}{\left(\mathrm{i} \omega+d_{z}\right)^{3}} \frac{\partial^{2} u}{\partial z \partial x}+\left(\frac{3(\mathrm{i} \omega)^{2} d_{z p}^{\prime 2}}{\left(\mathrm{i} \omega+d_{x}\right)^{4}}-\frac{(\mathrm{i} \omega)^{2} d_{z p p}^{\prime \prime}}{\left(\mathrm{i} \omega+d_{x}\right)^{3}}+\frac{\omega^{2}}{c^{2}}\right) \frac{\partial u}{\partial x}=-\frac{1}{c^{2}} \frac{\partial s}{\partial x},
\end{aligned}
$$

where $d_{x p}^{\prime}$ and $d_{x p p}^{\prime \prime}$ are the first-order and second-order derivatives of $d_{x}$.

Then, according to Yang et al., (2003, 2006), Tong $(2011,2013)$ and Lang and Yang, (2017), the discretization of each partial derivative using the NAD method is obtained as:

$$
\begin{gathered}
\frac{\partial^{2} u_{i, j, k}}{\partial x^{2}}=\frac{2}{\Delta x^{2}}\left(u_{i+1, j, k}-2 u_{i, j, k}+u_{i-1, j, k}\right)-\frac{1}{2 \Delta x}\left(\frac{\partial u_{i+1, j, k}}{\partial x}-\frac{\partial u_{i-1, j, k}}{\partial x}\right), \\
\frac{\partial^{2} u_{i, j, k}}{\partial y^{2}}=\frac{2}{\Delta y^{2}}\left(u_{i, j+1, k}-2 u_{i, j, k}+u_{i, j-1, k}\right)-\frac{1}{2 \Delta y}\left(\frac{\partial u_{i, j+1, k}}{\partial y}-\frac{\partial u_{i, j-1, k}}{\partial y}\right), \\
\frac{\partial^{2} u_{i, j, k}}{\partial z^{2}}=\frac{2}{\Delta z^{2}}\left(u_{i, j, k+1}-2 u_{i, j, k}+u_{i, j, k-1}\right)-\frac{1}{2 \Delta z}\left(\frac{\partial u_{i, j, k+1}}{\partial z}-\frac{\partial u_{i, j, k-1}}{\partial z}\right), \\
\frac{\partial^{3} u_{i, j, k}}{\partial x^{3}}=\frac{15}{2 \Delta x^{3}}\left(u_{i+1, j, k}-u_{i-1, j, k}\right)-\frac{3}{2 \Delta x^{2}}\left(\frac{\partial u_{i+1, j, k}}{\partial x}+8 \frac{\partial u_{i, j, k}}{\partial x}+\frac{\partial u_{i-1, j, k}}{\partial x}\right), \\
\frac{\partial^{3} u_{i, j, k}}{\partial y^{3}}=\frac{15}{2 \Delta y^{3}}\left(u_{i, j+1, k}-u_{i, j-1, k}\right)-\frac{3}{2 \Delta y^{2}}\left(\frac{\partial u_{i, j+1, k}}{\partial y}+8 \frac{\partial u_{i, j, k}}{\partial y}+\frac{\partial u_{i, j-1, k}}{\partial y}\right),
\end{gathered}
$$




$$
\frac{\partial^{3} u_{i, j, k}}{\partial z^{3}}=\frac{15}{2 \Delta z^{3}}\left(u_{i, j, k+1}-u_{i, j, k-1}\right)-\frac{3}{2 \Delta z^{2}}\left(\frac{\partial u_{i, j, k+1}}{\partial z}+8 \frac{\partial u_{i, j, k}}{\partial z}+\frac{\partial u_{i, j, k-1}}{\partial z}\right) .
$$

Taking the cross-derivative in $x$ direction as an example:

$$
\begin{aligned}
& \frac{\partial^{2} u_{i, j, k}}{\partial x \partial y}=\frac{1}{2 \Delta x \Delta y}\left(u_{i+1, j+1, k}+u_{i-1, j-1, k}-u_{i-1, j+1, k}-u_{i+1, j-1, k}\right) \\
& -\frac{1}{8 \Delta x}\left(\frac{\partial u_{i+1, j+1, k}}{\partial y}-\frac{\partial u_{i-1, j-1, k}}{\partial y}-\frac{\partial u_{i-1, j+1, k}}{\partial y}+\frac{\partial u_{i+1, j-1, k}}{\partial y}\right) \\
& -\frac{1}{8 \Delta y}\left(\frac{\partial u_{i+1, j+1, k}}{\partial x}-\frac{\partial u_{i-1, j-1, k}}{\partial x}-\frac{\partial u_{i+1, j-1, k}}{\partial x}+\frac{\partial u_{i-1, j+1, k}}{\partial x}\right), \\
& \frac{\partial^{2} u_{i, j, k}}{\partial x \partial z}=\frac{1}{2 \Delta x \Delta z}\left(u_{i+1, j, k+1}+u_{i-1, j, k-1}-u_{i-1, j, k+1}-u_{i+1, j, k-1}\right) \\
& -\frac{1}{8 \Delta x}\left(\frac{\partial u_{i+1, j, k+1}}{\partial z}-\frac{\partial u_{i-1, j, k-1}}{\partial z}-\frac{\partial u_{i-1, j, k+1}}{\partial z}+\frac{\partial u_{i+1, j, k-1}}{\partial z}\right) \\
& -\frac{1}{8 \Delta z}\left(\frac{\partial u_{i+1, j, k+1}}{\partial x}-\frac{\partial u_{i-1, j, k-1}}{\partial x}-\frac{\partial u_{i+1, j, k-1}}{\partial x}+\frac{\partial u_{i-1, j, k+1}}{\partial x}\right), \\
& \frac{\partial^{3} u_{i, j, k}}{\partial x^{2} \partial y}=\frac{5}{4 \Delta x^{2} \Delta y}\left(u_{i+1, j+1, k}+u_{i-1, j+1, k}-u_{i+1, j-1, k}-u_{i-1, j-1, k}+2 u_{i, j-1, k}-2 u_{i, j+1, k}\right) \\
& -\frac{1}{4 \Delta x \Delta y}\left(\frac{\partial u_{i+1, j+1, k}}{\partial x}+\frac{\partial u_{i-1, j-1, k}}{\partial x}-\frac{\partial u_{i-1, j+1, k}}{\partial x}-\frac{\partial u_{i+1, j-1, k}}{\partial y}\right) \\
& -\frac{1}{4 \Delta x^{2}}\left(\frac{\partial u_{i+1, j+1, k}}{\partial y}+\frac{\partial u_{i-1, j-1, k}}{\partial y}+\frac{\partial u_{i-1, j+1, k}}{\partial y}+\frac{\partial u_{i+1, j-1, k}}{\partial y}-2 \frac{\partial u_{i, j+1, k}}{\partial y}-2 \frac{\partial u_{i, j-1, k}}{\partial y}\right), \\
& \frac{\partial^{3} u_{i, j, k}}{\partial x^{2} \partial z}=\frac{5}{4 \Delta x^{2} \Delta z}\left(u_{i+1, j, k+1}+u_{i-1, j, k+1}-u_{i+1, j, k-1}-u_{i-1, j, k-1}+2 u_{i, j, k-1}-2 u_{i, j, k+1}\right) \\
& -\frac{1}{4 \Delta x \Delta z}\left(\frac{\partial u_{i+1, j, k+1}}{\partial x}+\frac{\partial u_{i-1, j, k-1}}{\partial x}-\frac{\partial u_{i-1, j, k+1}}{\partial x}-\frac{\partial u_{i+1, j, k-1}}{\partial y}\right) \\
& -\frac{1}{4 \Delta x^{2}}\left(\frac{\partial u_{i+1, j, k+1}}{\partial z}+\frac{\partial u_{i-1, j, k-1}}{\partial z}+\frac{\partial u_{i-1, j, k+1}}{\partial z}+\frac{\partial u_{i+1, j, k-1}}{\partial z}-2 \frac{\partial u_{i, j, k+1}}{\partial z}-2 \frac{\partial u_{i, j, k-1}}{\partial z}\right), \\
& \frac{\partial^{3} u_{i, j, k}}{\partial x \partial y^{2}}=\frac{5}{4 \Delta x \Delta y^{2}}\left(u_{i+1, j+1, k}+u_{i+1, j-1, k}-u_{i-1, j+1, k}-u_{i-1, j-1, k}+2 u_{i-1, j, k}-2 u_{i+1, j, k}\right) \\
& -\frac{1}{4 \Delta x \Delta y}\left(\frac{\partial u_{i+1, j+1, k}}{\partial y}+\frac{\partial u_{i-1, j-1, k}}{\partial y}-\frac{\partial u_{i+1, j-1, k}}{\partial y}-\frac{\partial u_{i-1, j+1, k}}{\partial y}\right) \\
& -\frac{1}{4 \Delta y^{2}}\left(\frac{\partial u_{i+1, j+1, k}}{\partial x}+\frac{\partial u_{i-1, j-1, k}}{\partial x}+\frac{\partial u_{i+1, j-1, k}}{\partial x}+\frac{\partial u_{i-1, j+1, k}}{\partial x}-2 \frac{\partial u_{i+1, j, k}}{\partial x}-2 \frac{\partial u_{i-1, j, k}}{\partial x}\right), \\
& \frac{\partial^{3} u_{i, j, k}}{\partial x \partial z^{2}}=\frac{5}{4 \Delta x \Delta z^{2}}\left(u_{i+1, j, k+1}+u_{i+1, j, k-1}-u_{i-1, j, k+1}-u_{i-1, j, k-1}+2 u_{i-1, j, k}-2 u_{i+1, j, k}\right) \\
& -\frac{1}{4 \Delta x \Delta z}\left(\frac{\partial u_{i+1, j, k+1}}{\partial z}+\frac{\partial u_{i-1, j, k-1}}{\partial z}-\frac{\partial u_{i+1, j, k-1}}{\partial z}-\frac{\partial u_{i-1, j, k+1}}{\partial z}\right) \\
& -\frac{1}{4 \Delta z^{2}}\left(\frac{\partial u_{i+1, j, k+1}}{\partial x}+\frac{\partial u_{i-1, j, k-1}}{\partial x}+\frac{\partial u_{i+1, j, k-1}}{\partial x}+\frac{\partial u_{i-1, j, k+1}}{\partial x}-2 \frac{\partial u_{i+1, j, k}}{\partial x}-2 \frac{\partial u_{i-1, j, k}}{\partial x}\right) .
\end{aligned}
$$

\section{References}

Bozdağ, E., Peter, D., Lefebvre, M., Komatitsch, D., Tromp, J., Hill, J., Podhorszki, N., and Pugmire, D. (2016). Global adjoint tomography: first-generation model. Geophys. J. Int., 207(3), 1739-1766.

https://doi.org/10.1093/gji/ggw356

Brossier, R., Operto, S., and Virieux, J. (2009). Seismic imaging of complex onshore structures by $2 \mathrm{D}$ elastic frequency-domain full-waveform inversion. Geophysics, 74(6), WCC105-WCC118. https://doi.org/10.1190/1.3215771

Day, S. M. (1982). Three-dimensional simulation of spontaneous rupture: the effect of nonuniform prestress. Bull. Seismol. Soc. Am., 72(6A), 1881-1902.

Dong, X. P., Yang, D. H., and Niu, F. L. (2019). Passive Adjoint tomography of the crustal and upper mantle beneath eastern Tibet with a W2-norm misfit function. Geophys. Res. Lett., 46(22), 12986-12995. https://doi.org/10.1029/2019GL085515

He, X. J., Yang, D. H., Huang, X. Y., and Ma, X. (2020). A numerical dispersion- dissipation analysis of discontinuous Galerkin methods based on quadrilateral and triangular elements. Geophysics, 85(3), T101-T121. https://doi.org/10.1190/geo2019-0109.1

Igel, H., Mora, P., and Riollet, B. (1995). Anisotropic wave propagation through finite-difference grids. Geophysics, 60(4), 1203-1216. https://doi.org/10.1190/1.1443849

Kelly, K. R., Ward, R. W., Treitel, S., and Alford, R. M. (1976). Synthetic seismograms: a finite-difference approach. Geophysics, 41(1), 2-27. https://doi.org/10.1190/1.1440605

Komatitsch, D., and Tromp, J. (2003). A perfectly matched layer absorbing boundary condition for the second-order seismic wave equation. Geophys. J. Int., 154(1), 146-153. https://doi.org/10.1046/j.1365-246X.2003.01950.x

Komatitsch, D., Tsuboi, S., and Tromp, J. (2005). The spectral-element method in seismology. In A. Levander, et al. (Eds.), Seismic Earth: Array Analysis of Broadband Seismograms, Volume 157 (pp. 205-227). Washington, DC: American Geophysical Union. https://doi.org/10.1029/157GM13 
Kosloff, D. D., and Baysal, E. (1982). Forward modeling by a Fourier method. Geophysics, 47(10), 1402-1412. https://doi.org/10.1190/1.1441288

Lailly, P. (1983). The seismic inverse problem as a sequence of before stack migration. In SIAM Conference on Inverse Scattering: Theory and Applications, Expanded Abstracts (pp. 206-220). SIAM.

Lang, C., and Yang, D. H. (2017). A nearly analytic discrete method for solving the acoustic-wave equations in the frequency domain. Geophysics, 82(1), T43-T57. https://doi.org/10.1190/geo2016-0248.1

Li, J. S., Yang, D. H., and Liu, F. Q. (2013). An efficient reverse time migration method using local nearly analytic discrete operator. Geophysics, 78(1), S15-S23. https://doi.org/10.1190/geo2012-0247.1

Liu, Q. Y., and Tromp, J. (2006). Finite-frequency kernels based on adjoint methods. Bull. Seismol. Soc. Am., 96(6), 2383-2397. https://doi.org/10.1785/0120060041

Liu, S. L., Li, X. F., Wang, W. S., Liu, Y. S., Zhang, M. G., and Zhang, H. (2014). A new kind of optimal second-order symplectic scheme for seismic wave simulations. Sci China Earth Sci, 57(4), 751-758. https://doi.org/10.1007/s11430-013-4805-0

Liu, S. L., Li, X. F., Wang, W. S., Xu, L., and Li, B. F. (2015). A modified symplectic scheme for seismic wave modeling. J. Appl. Geophys., 116, 110-120. https://doi.org/10.1016/j.jappgeo.2015.03.007

Lysmer, J., and Drake, L. A. (1972). A finite element method for seismology. Methods Comput. Phys.: Adv. Res. Appl., 11, 181-216. https://doi.org/10.1016/B978-0-12-460811-5.50009-X

Ma, X., Yang, D. H., He, X. J., Li, J. S., and Zheng, Y. C. (2018). A new high-order scheme based on numerical dispersion analysis of the wave phase velocity for semidiscrete wave equations. Geophysics, 83(3), T123-T138. https://doi.org/10.1190/geo2017-0441.1

Marfurt, K. J. (1984). Accuracy of finite-difference and finite-element modeling of the scalar and elastic wave equations. Geophysics, 49(5), 533-549. https://doi.org/10.1190/1.1441689

Mora, P. (1987). Nonlinear two-dimensional elastic inversion of multioffset seismic data. Geophysics, 52(9), 1211-1228. https://doi.org/10.1190/1.1442384

Nocedal, J., and Wright, S. J. (2006). Numerical Optimization (2nd ed). Berlin: Springer.

Operto, S., Ravaut, C., Improta, L., Virieux, J., Herrero, A., and Dell'Aversana, P. (2004). Quantitative imaging of complex structures from dense wideaperture seismic data by multiscale traveltime and waveform inversions: a case study. Geophys. Prosp., 52(6), 625-651. https://doi.org/10.1111/j.13652478.2004.00452.x

Operto, S., Virieux, J., Amestoy, P., L'Excellent, J. Y., Giraud., L., and Ben Hadj Ali, H. (2007). 3-D finite-difference frequency-domain modeling of viscoacoustic wave propagation using a massively parallel direct solver: a feasibility study. Geophysics, 72(5), SM195-SM211. https://doi.org/10.1190/1.2759835

Pan, W. Y., Innanen, K. A., Margrave, G. F., Fehler, M. C., Fang, X. D., and Li, J. X. (2016). Estimation of elastic constants for HTI media using Gauss-Newton and full-Newton multiparameter full-waveform inversion. Geophysics, 81(5), R275-R291. https://doi.org/10.1190/geo2015-0594.1

Pan, W. Y., Innanen, K. A., and Liao, W. Y. (2017). Accelerating Hessian-free Gauss-Newton full-waveform inversion via /-BFGS preconditioned conjugate-gradient algorithm. Geophysics, 82(2), R49-R64. https://doi.org/10.1190/geo2015-0595.1

Plessix, R. E. (2009). Three-dimensional frequency-domain full-waveform inversion with an iterative solver. Geophysics, 74(6), WCC149-WCC157. https://doi.org/10.1190/1.3211198

Pratt, R. G., and Worthington, M. H. (1990). Inverse theory applied to multisource cross-hole tomography. Part 1: acoustic wave-equation method. Geophys. Prosp., 38(3), 287-310. https://doi.org/10.1111/j.1365- 2478.1990.tb01846.x

Pratt, R. G., Shin, C., and Hick, G. J. (1998). Gauss-Newton and full Newton methods in frequency-space seismic waveform inversion. Geophys. J. Int., 133(2), 341-362. https://doi.org/10.1046/j.1365-246X.1998.00498.x

Pratt, R. G. (1999). Seismic waveform inversion in the frequency domain, Part 1: theory and verification in a physical scale model. Geophysics, 64(3), 888-901. https://doi.org/10.1190/1.1444597

Seriani, G., and Priolo, E. (1994). Spectral element method for acoustic wave simulation in heterogeneous media. Finite Elem. Anal. Des., 16(3-4), 337-348. https://doi.org/10.1016/0168-874X(94)90076-0

Sirgue, L., and Pratt, R. G. (2004). Efficient waveform inversion and imaging: a strategy for selecting temporal frequencies. Geophysics, 69(1), 231-248. https://doi.org/10.1190/1.1649391

Song, Z. M., and Williamson, P. R. (1995). Frequency-domain acoustic-wave modeling and inversion of crosshole data: part I-2.5-D modeling method. Geophysics, 60(3), 784-795. https://doi.org/10.1190/1.1443817

Tape, C., Liu, Q. Y., and Tromp, J. (2007). Finite-frequency tomography using adjoint methods-methodology and examples using membrane surface waves. Geophys. J. Int., 168(3), 1105-1129. https://doi.org/10.1111/j.1365246X.2006.03191.x

Tarantola, A. (1986). A strategy for nonlinear elastic inversion of seismic reflection data. Geophysics, 51(10), 1893-1903.

https://doi.org/10.1190/1.1442046

Tong, P., Yang, D. H., and Hua, B. L. (2011). High accuracy wave simulation-revised derivation, numerical analysis and testing of a nearly analytic integration discrete method for solving acoustic wave equation. International Journal of Solids and Structures, 48(1), 56-70. https://doi.org/10.1016/j.ijsolstr.2010.09.003

Tong, P., Yang, D. H., Hua, B. L., and Wang, M. X. (2013). A high-order stereomodeling method for solving wave equations. Bull. Seismol. Soc. Am., 103(2A), 811-833. https://doi.org/10.1785/0120120144

Tromp, J., Tape, C., and Liu, Q. (2005). Seismic tomography, adjoint methods, time reversal, and banana-doughnut kernels. Geophys. J. Int., 160(1), 195-216. https://doi.org/10.1111/j.1365-246X.2004.02453.x

Virieux, J., and Operto, S. (2009). An overview of full-waveform inversion in exploration geophysics. Geophysics., 74(6), WCC1-WCC26.. https://doi.org/10.1190/1.3238367

Wang, J., Yang, D. H., Jing H., and Wu, H. (2019). Full waveform inversion based on the ensemble Kalman filter method using uniform sampling without replacement. Sci. Bull., 64(5), 321-330. https://doi.org/10.1016/j.scib.2019.01.021

Yang, D. H., Teng, J. W., Zhang, Z. J., and Liu, E. R. (2003). A nearly analytic discrete method for acoustic and elastic wave equations in anisotropic media. Bull. Seismol. Soc. Am., 93(2), 882-890. https://doi.org/10.1785/0120020125

Yang, D. H., Lu, M., Wu, R. S., and Peng, J. M. (2004). An optimal nearly analytic discrete method for 2D acoustic and elastic wave equations. Bull. Seismol. Soc. Am., 94(5), 1982-1992. https://doi.org/10.1785/012003155

Yang, D. H., Peng, J. M., Lu, M., and Terlaky, T. (2006). Optimal nearly analytic discrete approximation to the scalar wave equation. Bull. Seismol. Soc. Am., 96(3), 1114-1130. https://doi.org/10.1785/0120050080

Yang, D. H., Wang, L., and Deng, X. Y. (2010). An explicit split-step algorithm of the implicit Adams method for solving 2-D acoustic and elastic wave equations. Geophys. J. Int., 180(1), 291-310. https://doi.org/10.1111/j.1365246X.2009.04407.x

Yang, D. H., Wang, N., and Liu, E. (2012). A strong stability-preserving predictorcorrector method for the simulation of elastic wave propagation in anisotropic media. Commun. Comput. Phys., 12(4), 1006-1032. https://doi.org/10.4208/cicp.010111.230911a 